\title{
Neural correlates of ataxia severity in spinocerebellar ataxia type 3/Machado- Joseph disease
}

\author{
Carlos R. Hernandez-Castillo ${ }^{1}$, Rosalinda Diaz², Aurelio Campos-Romo ${ }^{3}$ and Juan Fernandez-Ruiz²
}

\begin{abstract}
Background: Spinocerebellar ataxia type 3/Machado-Joseph disease (SCA3/MJD) is an autosomal dominant inherited neurodegenerative disorder. Several post-mortem and imaging studies have shown cerebellar and brainstem atrophy. A number of studies have used volumetric regional information to investigate the relationship between neurodegeneration and the ataxia severity. However, regional analysis can obscure the specific location in which the degenerative process is affecting the brain tissue, which can be crucial for the development of new target treatments for this disease.

Here we explored the relationship between the gray matter degeneration and the ataxia severity on a cohort of SCA3 patients using a voxel-wise approach.

Methods: Seventeen patients with molecular diagnose of SCA3 and 17 matched healthy controls participated in this study. Magnetic resonance imaging (MRI) brain images were acquired and voxel-based morphometry was used to obtain the grey matter volume of each participant. Ataxia severity in the patient group was evaluated using the scale for the assessment and rating of ataxia (SARA).
\end{abstract}

Results: Group comparison revealed significant atrophy in SCA3 including bilateral cerebellum, vermis, brainstem, and occipital cortex. Significant negative correlations between gray matter volume and SARA scores were found in the cerebellum and the cingulate gyrus.

Conclusions: These findings highlight the specific contribution of the cerebellum and the cingulate cortex to the ataxia deficits among the other regions showing neurodegeneration in SCA3 patients.

Keywords: Atrophy, Machado-Joseph, SARA, Spinocerebellar ataxia, VBM

\section{Background}

Spinocerebellar ataxia type 3/Machado-Joseph disease (SCA3/MJD) is an autosomal dominant inherited neurodegenerative disorder with a wide range of clinical manifestations [1]. SCA3/MJD is caused by an unstable CAG trinucleotide repeat expansion within the coding region of a gene located on chromosome 14q32.1 [2]. The predominant symptoms are progressive ataxia, ophthalmoplegia, spastic gait and peripheral neuropathy [1-3]. Previous morphometric SCA3/MJD MRI analysis revealed significant atrophy of the cerebellar hemispheres,

\footnotetext{
*Correspondence: jfr@unam.mx

${ }^{2}$ Departamento de Fisiología, Facultad de Medicina, Universidad Nacional Autonoma de México, UNAM, CP 04510 Coyoacán, Ciudad de México, México

Full list of author information is available at the end of the article
}

cerebellar vermis, pontine base, middle cerebellar peduncle, medulla oblongata, cervical spinal cord and enlargement of the fourth ventricle [3-9]. However, only few studies have analyzed the possible relationship between brain degeneration and the ataxia severity in this disease [7-9]. In those reports, researchers have used manually predefined anatomical landmarks to segment and calculate the total volume of different anatomical structures [7], automated region segmentation to calculate cortical thickness and volume [8] or atlas predefined regions to later group them into one single region [9]. Those analyses led to broad correlations between volume of the cerebellum, brainstem and other cortical regions and the ataxia impairment. 
Table 1 Demographics of the patient group

\begin{tabular}{lllll}
\hline ID & Gender & Age & Age at onset & SARA \\
\hline P01 & F & 49 & 39 & 26 \\
P02 & M & 58 & 47 & 19.5 \\
P03 & F & 45 & 25 & 26 \\
P04 & M & 37 & 32 & 12.5 \\
P05 & F & 35 & 33 & 6.5 \\
P06 & F & 23 & 20 & 6 \\
P07 & F & 42 & 35 & 12 \\
P08 & F & 24 & 20 & 9 \\
P09 & F & 18 & 16 & 2.5 \\
P10 & F & 45 & 39 & 8 \\
P11 & M & 43 & 40 & 14.5 \\
P12 & M & 34 & 29 & 18 \\
P13 & M & 59 & 49 & 19.5 \\
P14 & M & 33 & 28 & 9.5 \\
P15 & F & 40 & 30 & 20 \\
P16 & M & 56 & 46 & 14.5 \\
P17 & F & 46 & 41 & 1.5 \\
\hline
\end{tabular}

However, a more accurate mapping of the disease-related degeneration and its association with the symptoms is key for the development of possible treatments/therapies for this specific type of patients. Here, we assessed the ataxia severity and gray matter degeneration in a cohort of
SCA3/MJD patients by using the scale for the assessment and rating of ataxia (SARA) and whole brain voxel basedmorphometry (VBM) to find voxel-wise associations between brain atrophy and motor impairment.

\section{Methods \\ Participants}

The patient group consisted of seventeen patients with a molecular diagnosis of SCA3/MJD (10 female; righthanded; mean age/SD, 40.1/11.9 years, for more detailed information look at Table 1). Motor impairment was measured using SARA [10], which has eight items, including tests of gait, stance, sitting, and speech, as well as a finger-chase test, finger-nose test, fast alternating movements, and heel-shin test. The control group consisted of 17 healthy volunteers that were age and gender matched. All participants gave written, informed consent before entering the study. The procedures carried out were in accordance with the ethical standards of the committees on human experimentation of the Universidad Nacional Autonoma de Mexico.

\section{Image acquisition}

All images were acquired using a 32-channel quadrature head coil in a 3.0-T Achieva MRI scanner (Phillips Medical Systems, Eindhoven, The Netherlands). Foam-rubber cushion was used for fixing the head of the subject in place, so as to minimize any head movements. The high-

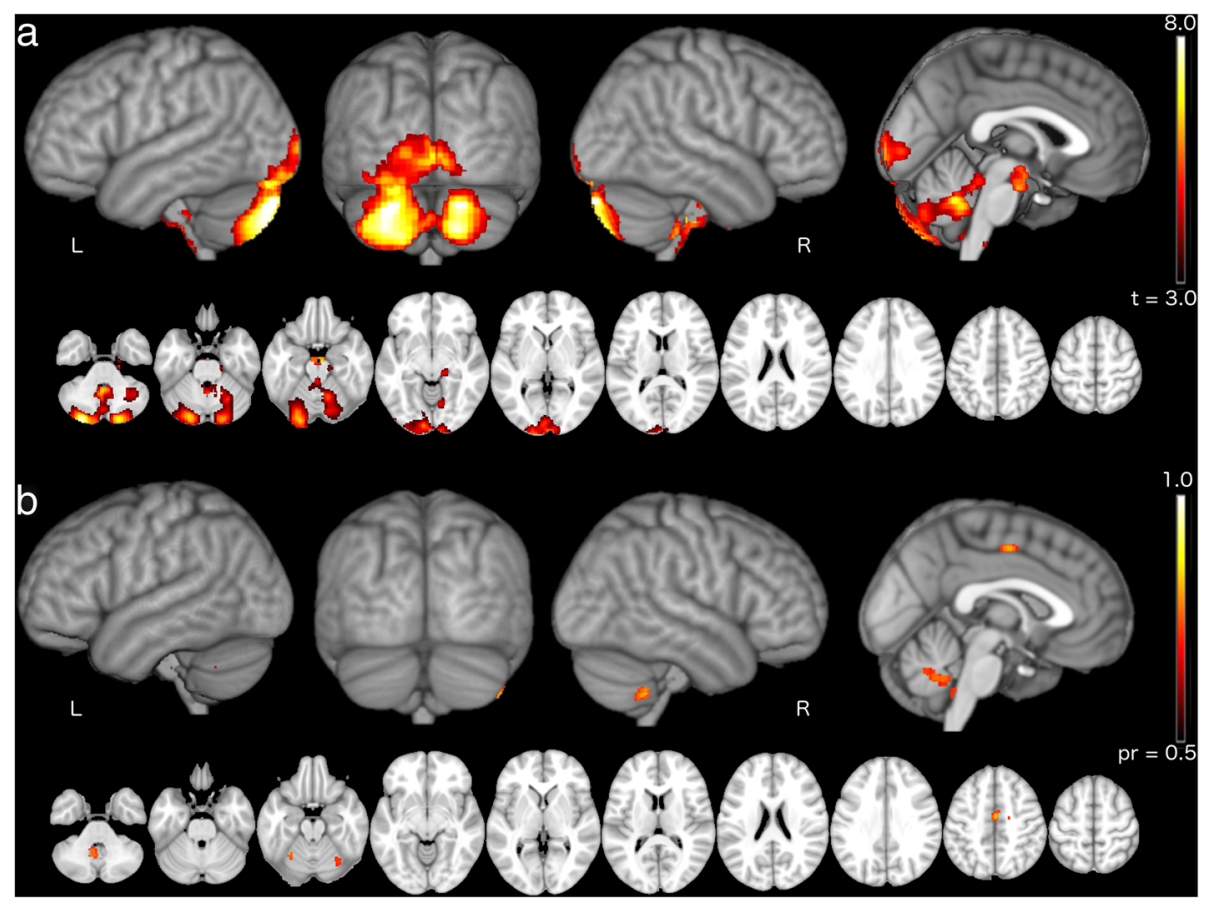

Fig. 1 Brain regions showing gray matter atrophy and SARA-GMV correlation. a Significant gray matter atrophy in patients compared with controls; b significant partial correlations between patients' GMV and SARA 
resolution anatomical acquisition consisted of a 3-D T1 Fast Field-Echo sequence, with TR/TE of $8 / 3.7 \mathrm{~ms}$, FOV of $256 \times 256 \mathrm{~mm}$, flip angle $25^{\circ}$ and an acquisition and reconstruction matrix of $256 \times 256$, resulting in an isometric resolution of $1 \times 1 \times 1 \mathrm{~mm}$.

\section{Voxel-based morphometry}

Gray matter volume (GMV) measurements were performed using voxel based morphometry (VBM) as implemented on FSL (FMRIB, Oxford, UK) following the standard procedure as previously reported [11]. Using the FSL randomise tool, a two-sample $t$ test was performed between the SCA3 group and controls. Significance was defined as $p<0.05$ after correcting for multiple comparisons using the randomized permutation method (TFCE). For the SCA3 group, whole-brain correlation maps were created by calculating the Pearson's partial correlation between the GMV and SARA scores including age in the analysis. Partial correlation maps were corrected for multiple comparisons by using false discovery rate (FDR) with a $p$ value $<0.05$.

\section{Results}

VBM analysis revealed gray matter atrophy in SCA3 patients compared to the control group (Fig. 1a) involving bilateral cerebellum, vermis, brainstem and the occipital cortex (Table 2). Four significant negative correlations were found between GMV and SARA scores in the SCA3 group (Fig. 1b) in regions of the cerebellum and the cingulate gyrus (Table 3 ).

\section{Discussion}

In this study, we analyzed the relationship between gray matter loss and SARA scores in SCA3 patients. As expected, significant negative correlations between SARA scores and GMV were found in the cerebellum, but also in the cingulate cortex.

Previous reports have shown the close relationship between the extent of the brain atrophy, predominately in the cerebellum, and a variety of symptoms in different

Table 2 Brain regions showing significant gray matter degeneration in SCA3/MJD patients

\begin{tabular}{llllll}
\hline Anatomical region & X & Y & Z & Cluster & t \\
\hline Left posterior cerebellum Crus II & -26 & -86 & -36 & 2633 & 7.73 \\
Right posterior cerebellum Crus II & 20 & -88 & -32 & 1419 & 7.31 \\
Right brainstem & 4 & -14 & -22 & 224 & 6.59 \\
Vermis IX & -2 & -54 & -34 & 114 & 6.45 \\
Pons & 2 & -14 & -24 & 219 & 6.19 \\
Right anterior cerebellum lobule V & 12 & -58 & -20 & 160 & 5.1 \\
Left lingual gyrus BA17 & -12 & -100 & -4 & 132 & 4.42 \\
Left anterior cerebellum lobule I-IV & -8 & -46 & -22 & 213 & 3.59 \\
\hline
\end{tabular}

Coordinates in MNI space in mm. BA=Brodmann Area
Table 3 Brain regions showing significant correlation between GMV and SARA

\begin{tabular}{llllll}
\hline Anatomical region & $\mathrm{X}$ & $\mathrm{Y}$ & $\mathrm{Z}$ & Cluster & $\mathrm{PC}$ \\
\hline Right cerebellum declive lobule VI & 32 & -64 & -14 & 191 & -0.825 \\
Left cerebellum culmen lobule VI & -28 & -50 & -18 & 180 & -0.816 \\
Left cerebellum tonsil lobule IX & -8 & -58 & -38 & 155 & -0.792 \\
Right paracentral lobule BA6 & 4 & -8 & 48 & 132 & -0.779 \\
\hline
\end{tabular}

Coordinates in $\mathrm{MNI}$ space in $\mathrm{mm}$. BA = Brodmann Area, $\mathrm{PC}=$ partial correlation

SCA subtypes [7-9, 12, 13]. Accordingly, our analysis showed a significant negative correlation between GMV and the SARA score in the bilateral lobule VI, extending to lobule $\mathrm{V}$, which are involved in sensorimotor processing as suggested by deficits in stroke patients [14, 15]. Lobule IX, which its GMV also correlated with SARA, is not only considered essential for visual guidance of movement [14], but its damage has been related to gait and balance impairment [15].

The only extra-cerebellar region where the GMV correlated with SARA score was the dorsal anterior cingulate, known to be critically involved in motor functions [16]. A previous report showed a significant degeneration in SCA3 in this area [6], however, our analysis also showed a negative correlation between GMV and the ataxia score, corroborating the functional relevance of this deterioration as shown by patients with lesions in this area, whom often show deficits in spontaneous initiation of movement and speech, as well as inability to suppress externally triggered motor subroutines [16].

\section{Conclusions}

In Conclusion, we report specific key areas where the GMV shows a close relationship with the ataxia impairment in SCA3. These findings add to previous reports $[7,9]$, while providing a more accurate localization of the SCA3/MJD ataxia-related areas.

\section{Abbreviations}

FDR: False Discovery Rate; GMV: Gray Matter Volume; MDJ: Machado-Joseph Disease; MRI: Magnetic Resonance Imaging; SARA: Scale for Assessing and Rating of Ataxia; SCA3: Spinocerebellar Ataxia 3; VBM: voxel-Based Morphometry

Acknowledgements

We want to thank all the patients and their caregivers for their participation in this study.

\section{Funding}

This study was founded in part by CONACYT 220871, PAPIIT IN214716 to JFR, and CONACYT 222009 to ACR

Availability of data and materials

Please contact the corresponding author for data requests.

\section{Authors' contributions}

CRHC designed the study Analyze the data and produced the first draft of the manuscript. JFR designed the study and provided expertise in brain imaging. RD and ACR were in charge of logistics as well as supervised and acquired data from patients. All authors read and approved the final manuscript. 


\section{Competing interest}

The authors declare that they have no competing interests.

\section{Consent for publication}

Not applicable.

\section{Ethics approval and consent to participate}

All participants gave written, informed consent before entering the study. The procedures carried out were in accordance with the ethical standards of the committees on human experimentation of the Universidad Nacional Autonoma de Mexico.

\section{Publisher's Note}

Springer Nature remains neutral with regard to jurisdictional claims in published maps and institutional affiliations.

\section{Author details}

${ }^{1}$ CONACYT - Instituto de Neuroetología - Universidad Veracruzana, Xalapa, Ver, Mexico. ${ }^{2}$ Departamento de Fisiología, Facultad de Medicina, Universidad Nacional Autonoma de México, UNAM, CP 04510 Coyoacán, Ciudad de México, México. ${ }^{3}$ Unidad Periférica de Neurociencias, Facultad de Medicina, Universidad Nacional Autónoma de México, en el Instituto Nacional de Neurología y Neurocirugía "Manuel Velasco Suárez", Ciudad de México, Mexico.

Received: 18 May 2017 Accepted: 29 May 2017

Published online: 06 June 2017

\section{References}

1. Matilla T, McCall A, Subramony SH, Zoghbi HY. Molecular and clinical correlations in spinocerebellar ataxia type 3 and Machado-Joseph disease. Ann Neurol. 1995;38(1):68-72.

2. Kawaguchi Y, Okamoto T, Taniwakiz M, Aizawa M. CAG expansions in a novel gene for Machado-Joseph disease at. Nat Genet. 1994;8(3):221-8.

3. Bürk K, Abele M, Fetter M, Dichgans J, Skalej M, Laccone F, Didierjean O, Brice A, Klockgether T. Autosomal dominant cerebellar ataxia type I clinical features and MRI in families with SCA1, SCA2 and SCA3. Brain. 1996;119(5):1497-505.

4. Lukas C, Schöls L, Bellenberg B, Rüb U, Przuntek H, Schmid G, Köster O, Suchan B. Dissociation of grey and white matter reduction in spinocerebellar ataxia type 3 and 6: a voxel-based morphometry study. Neurosci Lett. 2006;408(3):230-5.

5. Goel G, Pal PK, Ravishankar S, Venkatasubramanian G, Jayakumar PN, Krishna N, Purushottam M, Saini J, Faruq M, Mukherji M, Jain S. Gray matter volume deficits in spinocerebellar ataxia: an optimized voxel based morphometric study. Parkinsonism Relat Disord. 2011:17(7):521-7.

6. Guimarães RP, D'Abreu A, Yasuda CL, França MC, Silva BH, Cappabianco FA, Bergo FP, Lopes-Cendes IT, Cendes F. A multimodal evaluation of microstructural white matter damage in spinocerebellar ataxia type 3. Mov Disord. 2013;28(8):1125-32.

7. Schulz JB, Borkert J, Wolf S, Schmitz-Hübsch T, Rakowicz M, Mariotti C, Schoels L, Timmann D, van de Warrenburg B, Dürr A, Pandolfo M. Visualization, quantification and correlation of brain atrophy with clinical symptoms in spinocerebellar ataxia types 1, 3 and 6. Neuroimage. 2010;49(1):158-68.

8. Rezende TJ, D'abreu A, Guimaraes RP, Lopes TM, Lopes-Cendes I, Cendes F, Castellano G, França MC. Cerebral cortex involvement in Machado - Joseph disease. Eur J Neurol. 2015;22(2):277-e24.

9. D'Abreu A, Franca Jr MC, Yasuda CL, Campos BA, Lopes-Cendes I, Cendes F. Neocortical Atrophy in Machado-Joseph Disease: A Longitudinal Neuroimaging Study. J Neuroimaging. 2012;22(3):285-91.

10. Schmitz-Hübsch T, Du Montcel ST, Baliko L, Berciano J, Boesch S, Depondt C, Giunti P, Globas C, Infante J, Kang JS, Kremer B. Scale for the assessment and rating of ataxia Development of a new clinical scale. Neurology. 2006:66(11):1717-20.

11. Ashburner J, Friston KJ. Voxel-based morphometry-the methods. Neuroimage. 2000;11(6):805-21.

12. Hernandez-Castillo CR, Galvez V, Diaz R, Fernandez-Ruiz J. Specific cerebellar and cortical degeneration correlates with ataxia severity in spinocerebellar ataxia type 7. Brain Imaging Behav. 2016;10(1):252-7.

13. Reetz K, Lencer R, Hagenah JM, Gaser C, Tadic V, Walter U, Wolters A, Steinlechner S, Zühlke C, Brockmann K, Klein C. Structural changes associated with progression of motor deficits in spinocerebellar ataxia 17. The Cerebellum. 2010;9(2):210-7.

14. Stoodley CJ, Schmahmann JD. Evidence for topographic organization in the cerebellum of motor control versus cognitive and affective processing. Cortex. 2010;46(7):831-44.

15. Ilg W, Giese MA, Gizewski ER, Schoch B, Timmann D. The influence of focal cerebellar lesions on the control and adaptation of gait. Brain. 2008;131(11):2913-27.

16. Paus TS. Primate anterior cingulate cortex: where motor control, drive and cognition interface. Nat Rev Neurosci. 2001;2(6):417-24.

\section{Submit your next manuscript to BioMed Central and we will help you at every step:}

- We accept pre-submission inquiries

- Our selector tool helps you to find the most relevant journal

- We provide round the clock customer support

- Convenient online submission

- Thorough peer review

- Inclusion in PubMed and all major indexing services

- Maximum visibility for your research

Submit your manuscript at www.biomedcentral.com/submit
Biomed Central 